\title{
Correlative Analysis of Polymer Materials using Low Voltage and Variable Pressure SEM
}

\author{
S.J. Okerstrom* and K.X. Pham* \\ *Medtronic Inc. \\ 7000 Central Ave. NE \\ Minneapolis, MN 55432
}

Gold / palladium, platinum and carbon coating are commonly used on polymers, ceramics, biological materials and other non-conducting samples in the scanning electron microscope (SEM). They serve to conduct away electrons that otherwise would charge the surface of the sample. Both positive and negative charging result in gross distortion of the surface making the sample unviewable. The conductive coatings allow the microscopists to easily view non-conductive samples. However, several problems exist with coatings. First, the sample is exposed to energy / heat that may damage the surface or produce artifacts. Second, if one is performing microanalysis, the coating may mask the surface composition or overlap with the peaks of the elements in the sample.

In recent years one of two techniques has been used to image these samples in their natural state. They are low voltage or variable pressure scanning electron microscopy. Low voltage (LV) minimizes charge by reducing the voltage which reduces the beam energy and depth of penetration into the sample. Voltages of $5 \mathrm{KV}$ to $100 \mathrm{~V}$ are used in tungsten filament or field emission SEM. Variable pressure or "leaky vacuum" bleeds in a small amount of air increasing the pressure in the chamber to tens or hundreds of Pascals. These air molecules ionize conducting away the charge on the sample surface. Variable pressure (VP) technique uses a higher accelerating voltage of 15 to 20 $\mathrm{KV}$ and typically a backscattered detector.

We have found that these techniques should be used in tandem for many analyses of non-conductive samples to gain a more complete understanding of the materials. One example which illustrated the types of information gained from using both of the techniques is composite tubing with radiopaque particles mixed in the outside surface to make it visible in fluoroscopy. Figure 1 shows an image of the end of composite tubing examined in both LV and VP modes. The radiopaque particles appear bright in the VP mode using a backscattered detector.

Another example of examination of a surface treatment highlights the differences in depth of penetration between the two techniques. In figure 2, LV and VP images of same area of the surface are shown from an In Vivo study of a textured polymer. The VP image shows the full depth of the surface topography as the electrons penetrate more deeply into the material. Figure 3 VP image shows the treated surface of lead insulation. The texture allows it to slide more easily into the veins of the heart during implantation of a pacemaker. Figure 4 highlights the compositional difference in a study of steroid loading in a polymer ring.

These and other examples will illustrate the need for use of both techniques to gain complementary information in analysis of non-conductive uncoated polymers and other samples. 

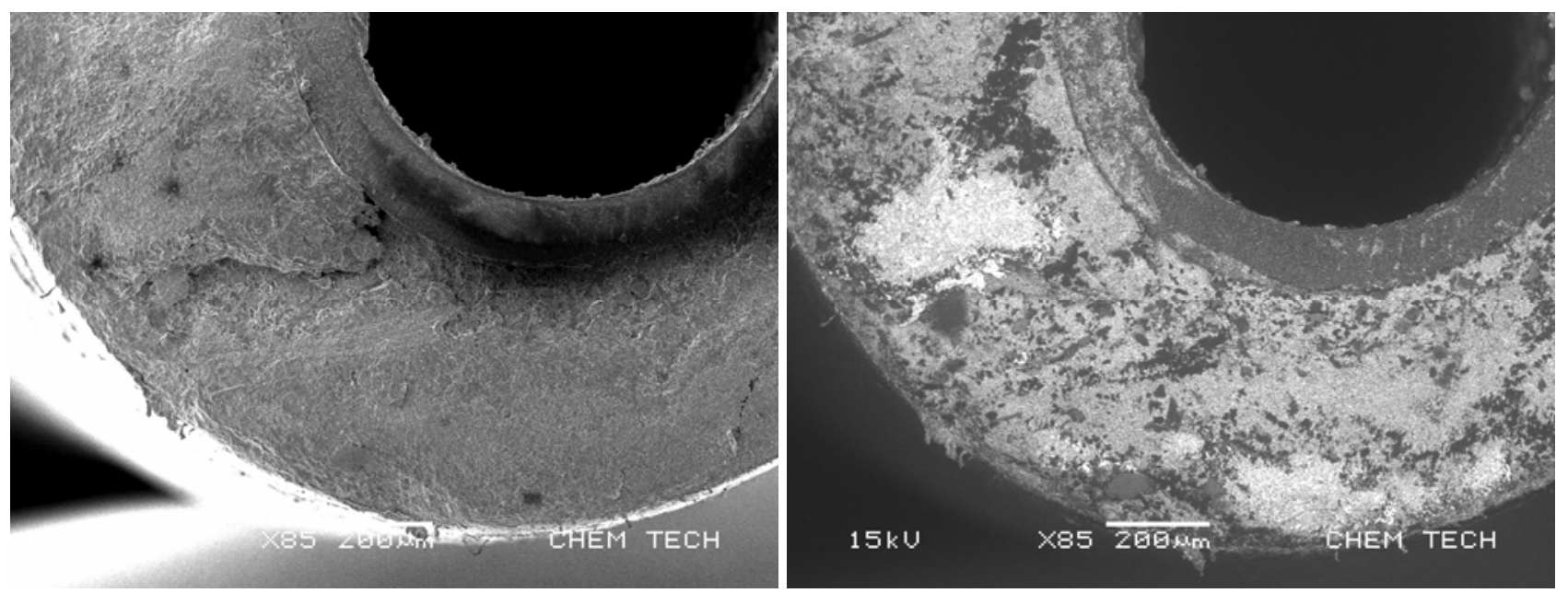

Fig. 1 LV (left) and VP (right) images of composite tubing with radiopaque particles in outer tube
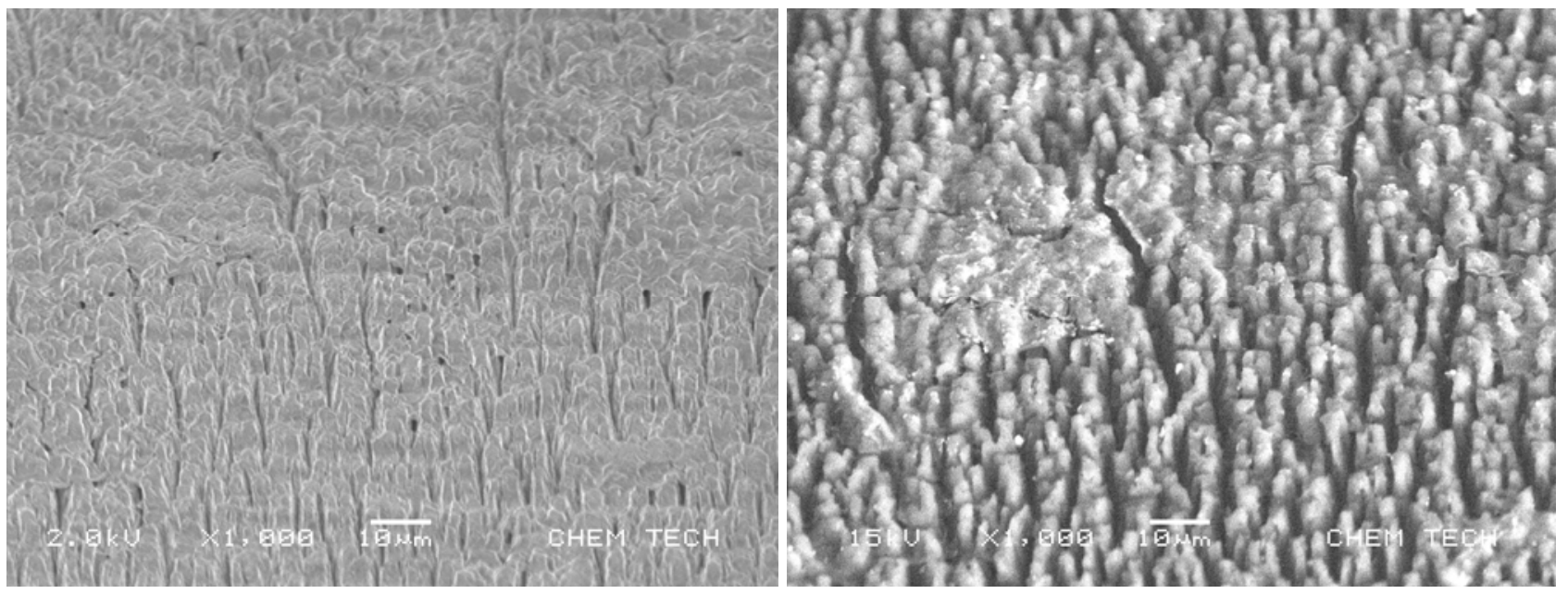

Fig. 2 LV (left) and VP (right) images of same area of textured polymer material from In Vivo Study

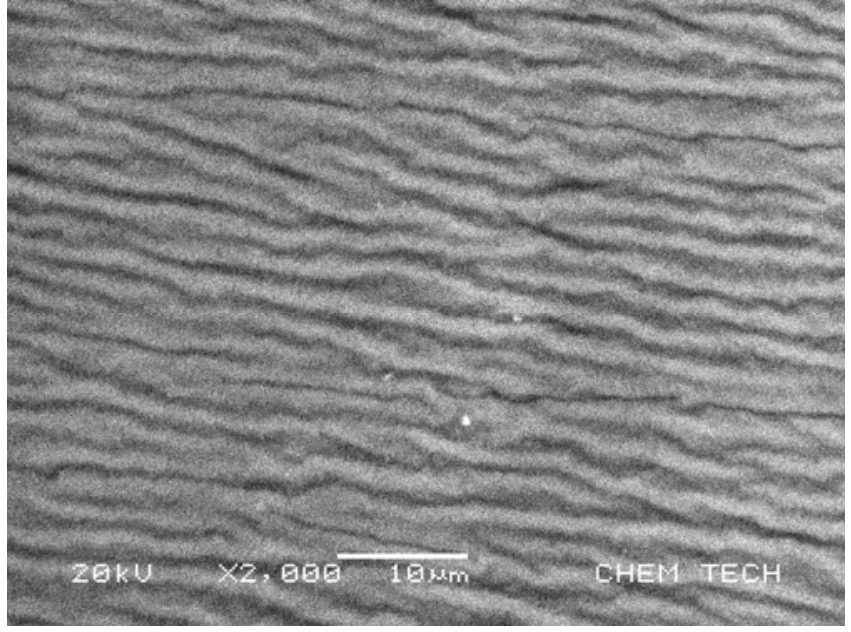

Fig. 3 VP BCI image of treated polymer tubing

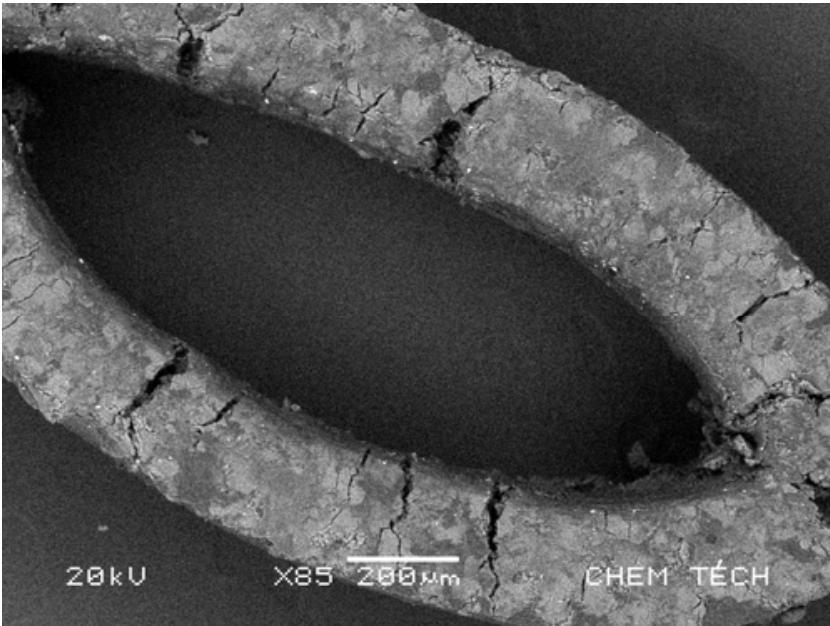

Fig. 4 VP image of cracking in steroid loaded ring 